\title{
The Collision of the Novel Corona Virus (COVID-19) Pandemic on Psychological Wellbeing and Mental Health
}

\author{
Yordanos Yibeltal Yedemie \\ Bahir Dar University, College of Education and Behavioral Sciences
}

\begin{abstract}
The unpredictable and uncertain COVID-19 incidence has the potential of adversely upsetting the psychological wellbeing on individual and community level. At present all efforts are listening carefully on the understanding of epidemiology, clinical diagnosis and prognosis, means of transmission, frustrate the spread of the virus, and challenges of worldwide physical condition, while crucially significant mental wellbeing has been unnoticed in this attempt. COVID-19 is leading to severe psychosocial panics and comprising mental health marking a secondary health concern all about the globe. Internationally implementing preventive and controlling measures, and humanizing coping and resilience are demanding factors; modified lifestyle; conspiracy theories, propaganda and disinformation about the origin, symptoms, transmission, prevention and treatment; international socioeconomic catastrophe; travel restrictions; workplace risk manage; postponement and annulment of religious, cultural and entertainment events, and overwhelmed health centers/institutes. Psychological intervention can be conducted in different ways to improve psychological hurting and pick up the treatment effect.
\end{abstract}

Keywords: COVID-19, pandemic, Psychological Wellbeing, Mental Health

DOI: $10.7176 /$ RHSS/10-15-01

Publication date: August $31^{\text {st }} 2020$

\section{Introduction}

WHO declared the corona virus disease 2019 (COVID-19) outbreak, caused by severe acute respiratory syndrome corona virus 2, to be a pandemic on March 12, 2020.1 On March 18, 2020, the UN Educational, Scientific and Cultural Organization estimated that 107 countries had implemented national school closures related to COVID-19, affecting 862 million children and young people, roughly half the global student population (Verity et al., 2020).

The occurrence has the potential for greater loss of life, significant disruptions in worldwide supply chains, lower commodity prices, and economic losses in both developed and developing countries. The COVID-19 outburst is affecting provide chains and disrupting manufacturing operations around the planet. Economic motion has fallen in the past 3 months, particularly in developed country, and is expected to remain depressed for months. The eruption is taking place at a time when universal economic activity is facing indecision and governments have limited policy space to do something. The duration and harshness of impacts of the COVID19 outbreak will depend on the projected distance end to end and location(s) of the outbreak, as well as on whether there are is a concerted, fast track response to support developing countries, where wellbeing systems are often weaker. With proactive repression measures, the loss of life and economic crash of the epidemic could be under arrest. It is hence dangerous for the global community to work together on the fundamental factors that are enabling the outbreak, on supporting policy responses, and on intensification response competence in developing countries - where health systems are weakest, and therefore populations most susceptible(CDCP, 2020).

The Collision of the Novel Corona Virus (COVID-19) Pandemic on Psychological Wellbeing and Mental Health \& suggestion ideas

In organizing psychological intervention within and across various stages of the pandemic, I want to emphasize most important challenges with recommended ideas:

1. Community underestimation of the psychological consequences of pandemics and, consequently, limited resources to cope with them. There is evidence that individuals exposed to public health emergencies have increased psychopathological vulnerability both during and after the potentially traumatic event. Even if the international COVID-19 pandemic response has been unparalleled in terms of mobilization of resource and finance, there will also be long-term impacts in terms of treatment burden, including psychological health, particularly in low resource and conflict settings. In my indulgent, the progression of COVID-19 provoked the psychological wellbeing of infected patients, the general population and health professionals. Therefore, it is important to evaluate and identify all risk groups and adapt interventions to their specific needs. Among the variables to consider are disease trajectory, severity of clinical symptoms, place of treatment (in-home or out-of-home isolation), and history of previous trauma and, previous history of mental health problems. Having this information will help categorize people at risk and enable specific preventive mental/psychological health measures to be put in place. 
2. Psychological Health care system discrepancies, both in terms of material and human resources or in mental health professionals not specialized in the psychological approach of crises and emergencies. In understanding, the shortage of human resources led to individual professionals accumulating multiple responsibilities, reducing the effectiveness of their interventions. For this reason, government, policy makers and health managers need to be aware of health systems strengthening for increasing the capacity of mental health professionals, facilitate training for emergency intervention, and monitor workload burdens.

3. Underprivileged preparation and coordination of psychological interventions, especially when they are applied at different levels and by different professionals. In my view, at the start of the COVID-19 outbreak, the absence of ample preparation of psychological interventions led to disorganized implementation, compromising effectiveness and efficacy, and hampering access to available health resources. Any psychological intervention should be planned and coordinated together with all the social-health stakeholders involved. This maximized the potential for ample continuity of care even after acute phase of the pandemic recedes.

4. There is too a risk attached to early crisis responses, leading to a propagation of interventions and frameworks associated with an oversupply of well-intentioned but potentially non-evidence based psychological assistance, often non-governmental organizations and the third sector. This is not to say all NGO interventions are compromised, and indeed prevention in mental health is highly desirable. That said, delivery of preventive interventions must be balanced by delivery and/or supervision applied by appropriately qualified professionals.

5. Therapeutic focus only self-regulation and overcoming distress or exploration of opportunities for building healthy parts and pursuing autonomy, exploration and expanding the healthy self? I have noted that in majority of cases where I have attuned delivery of psychotherapy to fit the pandemic restrictions, patients are seeking a balance between acceptance of the current situation, even as still trying to challenge maladaptive schemas and develop an emergent healthy part of the self. Certainly, once issues connecting to the present crisis have been dealt with, patient and therapist may explore how the current distressing conditions create suffering not only for their direct traumatic effects, but also because they may indirectly bring undermine existing personality, cognitive and emotional vulnerabilities to the fore. Therefore, professionals may help the patients connect their present experiences to lifelong vulnerabilities, enabling therapeutic work to continue as they did before the emergency, albeit with specific adaptations. For example, prior to lockdown patients with avoidant personality disorders may have started questioning schemas of them as inferior and others are judging and therefore, they coped with social avoidance. In this instant behavioral experiments aimed at increasing social contact and thus further challenging the schemas are more difficult to enact. Yet, the professionals may still explore opportunities, and build more basic steps for future real-life exposures. Patients looking for employment may be able to access online courses or training for life after. Patients searching for romantic partner may use dating Apps or explore the feelings and thoughts they experience when chatting with some new acquaintance.

6. Finally, the consequences of quarantine on psychological well-being should be discussed. And it is extremely necessary to implement public mental health policies in combination with epidemic and pandemic response strategies before, during and after the event.

As a Psychologist, I consider it essential to pay concentration to these factors so I can provide timely and appropriate mental health care directed to the needs of these different groups a challenge for the near future.

\section{REFERENCES}

Centers for Disease Control and Prevention (CDCP) (2020). Coronavirus disease 2019 (COVID-19) Retrieved on March 26, 2020 from https://www.cdc.gov/coronavirus/about/index.html

Legido-Quigley, H., Mateos-García, J. T., Campos, V. R., Gea-Sánchez, M., Muntaner, C., y McKee, M. (2020). The resilience of the Spanish health system against the COVID-19 pandemic. https://doi.org/10.1016/S2468-2667(20)30060-8

Yao H, Chen JH, Xu YF. Patients with mental health disorders in the COVID-19 epidemic. 2020;7:e21. [ Links ]

Verity, R., Okell, L. C., Dorigatti, I., Winskill, P., Whittaker, C., Imai, N., ... Ferguson, N. (2020). Estimates of the severity of COVID-19 disease. 